de Barthe et Dofilho. Ce rapport est au minimum égal à 1,62 pour les vaches hollandaises et à 1,72 pour les vaches suisses.

Si le lait est mouillé et salé simultanément, le dénominateur de la fraction augmente alors que le numérateur diminue ; pour ces deux raisons le rapport s'abaisse au-dessous des limites inférieures indiquées ; la fraude peut être dépistée.

Le chlore étant un élément uniquement minéral, il serait peutêtre plus justifié de ne le comparer qu'au phosphore minéral. Nos résultats montrent que le rapport $\frac{\mathrm{P} \text { minéral }}{\mathrm{Cl}}$ suit les mêmes variations que le rapport $\frac{\mathrm{P} \text { total }}{\mathrm{Cl}}$; en pratique, on peut se contenter de déterminer ce dernier rapport, le dosage du phosphore total étant plus rapide et plus simple que celui du phosphore minéral.

\title{
L'INFECTION LATENTE DE LA MAMELLE ET SES RÉVEILS. LES MOYENS DE LA DÉPISTER
}

\author{
par CH. PORCHER
}

Docteur is Sciences physiques.

(Suite)

Cette observation importante étant faite, il nous reste à donner maintenant quelques précisions sur l'allure des modifications chimiques enregistrées dans les laits malades.

On sait depuis longtemps que la constance du point de congélation du lait $(\Delta)$, traduisant la conservation de l'isotonie de cette sécrétion, s'observe aussi bien à l'état pathologique qu'à l'état normal. Du moins les variations du $\Delta$ ne sont pas à ce point considérables qu'elles nous interdisent de dire que la cellule mammaire frappée par l'infection, ou ayant à se soumettre à un autre processus (la rétention par exemple), porte atteinte à la concentration de son protoplasma en modifiant ses échanges nutritifs.

En 1906, nous avons le premier montré [34] que la conservation de l'isotonie de la sécrétion lactée dans les circonstances les plus variées, à l'état normal, au cours de la rétention ou pendant l'évolution des mammites, était le fait principal d'un jeu de bascule entre le lactose et le chlorure de sodium, par conséquent entre deux molécules de signification physiologique très différente : le lactose, molécule élaborée, et le chlorure de sodium, molécule non élaborée. Puisque l'abaissement cryoscopique du lait ne varie guère, que le lait soit normal ou malade, cette donnée physico-chimique ne peut pas être utilisée pour le diagnostic de l'état du lait. 
LES LAITS SALÉs. - Mais il n'en est pas de même - comme nous allons le voir - des autres données du même ordre. Retenons cependant ce fait important que, - la conservation de la valeur de $\Delta$ dépendant du jeu inverse du sucre et du sel : moins de suore et davantage de sel, - il va s'en suivre une modification de la saveur du lait; et, de fait, c'est une connaissance depuis longtemps acquise que les laits malades ont une saveur salée.

Nous avons indiqué plus haut que si objectivement le lait malade était apparemment normal par son aspect, sa couleur, son odeur, une réserve était à faire au sujet de sa saveur. Il faut déjà que l'infection latente ait nettement marqué son empreinte sur le lait pour que nous enregistrions cette modification de saveur. La diminution du sucre et l'augmentation du sel s'associent pour modifier la saveur originelle du lait normal. Quelquefois même la saveur salée dans les cas de mammite plus accentuée, tout en étant très franche, est accompagnée d'une légère amertume, due probablement à des phénomènes protéolytiques qui ont mis en liberté des protéoses, voire des peptones.

LA Rétention en face de L'infection. - En tout cas, c'est une notion de première importance qui doit vous guider dans votre diagnostic. Tout lait salé est un lait suspect. L'infection est là, à condition, bien entendu, que vous ayez éliminé au préalable l'influence dominante de la rétention. Il est évident que si vous êtes appelé à intervenir chez un animal en pleine lactation dont la sécrétion d'un ou deux quartiers a baissé sans raisons plausibles, le processus de la rétention ne joue point ici comme il le fait plus tard, lorsque régulièrement, à la fin d'une lactation, on assèche la glande.

Nous avons déjà appelé votre attention sur les relations de l'infection et de la rétention. Nous tenons à insister encore sur ce que les modifications chimiques, cytologiques et diastasiques qui se passent dans un lait de rétention sont tout à fait analogues, l'espèce et le taux microbiens étant laissés de côté, à celles que nous relevons dans un lait de mammite.

Il importe donc d'éliminer toute intervention possible de la rétention ou, du moins, lorsqu'il n'est pas possible de la négliger, d'en apprécier l'importance, d'en faire la part. Cela n'est pas toujours très facile : l'examen raisonné des circonstances, le dépouillement critique des symptômes peuvent toutefois vous aider.

Un lait de rétention devient salé comme un lait de mammite ; mais, pour en revenir au cas de tout à l'heure et qui est vraiment pathologique : celui de la diminution, sans raisons apparentes, de la quantité de lait donnée par un ou deux quartiers chez un même animal, il n'y a pas de rétention nettement dessinée à incriminer. On a, en effet, procédé à la traite comme d'habitude et le seul fait qu'on 
ait relevé, c'est une diminution du lait. Sans aucun doute, l'infection est déjà installée, la cellule mammaire est troublée dans son fonctionnement. Elle donne moins de lactose, mais elle laisse passer plus de sel:

Il est connu qu'en fin de lactation, alors que la glande mammaire n'est pas encore "asséchée ", certains quartiers qui fonctionnent au ralenti ont plus que d'autres tendance à donner du lait salé. Ici, nous avons l'association parallèle de deux jeux différents : celui de la rétention que détermine l'espacement des traites et celui de l'infection relevant du fait que lesdits quartiers possédaient antérieurement, bien qu'apparemment normaux, un taux microbien particulièrement élevé.

Le lait salé est riche en leucocytes. - Les auteurs qui ont travaillé sur le sujet que nous exposons ont constaté qu'un lait salé était en même temps un lait chargé de leucocytes. Tout cela se tient en effet et vous allez facilement comprendre pourquoi.

LE JEU des MOLÉcules ÉLABorées est INVERSE de celui des MOLÉCulES NON ÉLABORÉES. - L'exposé des troubles chimiques du lait au cours des mammites serait incomplet si nous nous limitions aux relations étroites qui existent entre le lactose et le chlorure de sodium.

Le lactose n'est pas la seule molécule qui soit élaborée. Il y a les citrates, il y a les phosphates. Ceux-ci et ceux-là sont touchés comme lui et sensiblement dans les mêmes proportions. Il y a également la caséine, et enfin, de même que nous avons vu le taux du chlorure de sodium, molécule non élaborée, s'élever lorsque celui du lactose s'abaisse, nous verrons également s'élever, et plus ou moins parallèlement, le taux des autres molécules non élaborées : azote non protéique, globuline, albumine, etc. Tout cela va nous permettre d'assurer comme une liaison entre les diverses faces de l'aspect chimique d'un lait altéré du fait de l'infection.

La diminution du lactose est parallèle à celle de l'acide phosphorique et de la potasse. A la diminution des phosphates potassiques répond une baisse de l'acidité ionique. Toutes les fois que le lactose baisse, nous trouvons que :

$1^{\circ}$ L'acide phosphorique baísse ;

$2^{\circ}$ La potasse baisse.

L'abaissement du taux de l'acide phosphorique et de la potasse relève d'une diminution des phosphates mono- et bipotassiques. Or, au phosphate monopotassique appartient presque en son entier l'acidité du lait, l'acidité ionique, celle qui est décelée par le potentiomètre 
ou par des indicateurs colorés sensibles qui ont pénétré sur le terrain de la biologie au cours de ces dernières années.

En même temps, et évidemment, l'acidité de titration par la soude en présence de la phtaléine du phénol diminue également, mais pas toujours dans les mêmes proportions, parce que sa signification est tout autre que celle de l'acidité ionique.

Un lait riche en matières protéiques : caséine, albumine, globuline, peut avoir une acidité de titration assez élevée, alors que son acidité ionique est restée normale et se trouve quelquefois même abaissée.

La mesure de l'acidité du lait est done très importante dans la détection des mammites. Ce n'est pas l'acidité de titration que nous vous recommanderons de mesurer systématiquement, mais bien l'acidité ionique, c'est-à-dire le $p H$ qui lui correspond ; nous verrons tout à l'heure quels sont les indicateurs colorés qu'il faut prendre et dans quelles conditions on doit les employer.

Quand le lactose baisse, nous trouvons encore que :

$3^{\circ}$ Les citrates baissent. Leur taux diminue considérablement et dans les laits malades il n'y a plus d'acide citrique du tout [35].

$4^{\circ}$ La caséine baisse également et, en même temps qu'elle, les phosphates de calcium qui lui sont unis dans le complexe caséinate de calcium + phosphates de calcium, système tout à fait caractéristique de la sécrétion lactée et sur lequel porte essentiellement l'action de la présure.

L'aspect de colle de certains laits mammiteux. - Au complexe, notamment aux phosphates de calcium, est due principalement la blancheur du lait. Les deux éléments du complexe viennent-ils à diminuer par suite de l'infection, le lait tend à prendre l'apparence d'une colle plus ou moins translucide. C'est celle que nous observons dans la plupart des laits malades assez altérés ; c'est celle qui caractérise le "Gelber Galt" des Allemands. Nous avons là un élément d'appréciation qu'il ne faut pas négliger. C'est aussi cette apparence de colle que nous saisirons dans les laits colostraux et dans les laits de rétention.

Les laits de mammite coagulent moins bien par la présure. - De la diminution du taux du caséinate et de celui des phosphates de calcium, il résulte que la réaction du lait vis-à-vis de la présure sera moins vigoureuse que celle du lait normal. Un lait normal caille après l'addition de lab-ferment en donnant un coagulum ferme, élastique, ayant le même volume que le lait initial et dont la synérèse s'effectue normalement. Un lait malade donne au contraire un caillé mou, fragmenté, se détachant aisément des parois 
du récipient, nageant au milieu d'un sérum trouble. SchERN [36] a bien étudié cet aspect de la question.

Les laits de mammites et l'industrie fromagère. - Le préjudice causé à l'industrie fromagère par la mauvaise qualité du caillé d'emprésurage s'explique par ce qui vient d'être dit. Mais ce qui est grave, c'est qu'il suffit parfois d'une petite quantité d'un lait malade pour gêner l'emprésurage d'une masse importante de lait normal dans lequel il se trouve dilué. Puisqu'on ne peut, dans ces conditions, invoquer une diminution sensible des phosphates de chaux et de la caséine dans le lait moyen de la cuve, on doit se demander comment l'emprésurage aboutit à de si mauvais résultats. Le lait malade apporte avec lui un taux d'albumine et surtout de globuline assez élevé. Les recherches que nous avons faites dans notre laboratoire sur le lait de femme, avec la collaboration de Mue J. BRIGANDO, - recherches qui ne sont pas encore publiées, mais dont l'essentiel a été, en ce qui concerne le point que nous étudions ici, donné dans un travail préliminaire [37], - nous ont montré que la globuline, par le rôle protecteur qu'elle assume vis-à-vis des autres protéines du lait, principalement du caséinate de calcium et par suite du complexe, nuit à la coagulation par la présure. Done, par sa présence et alors que ses proportions en sont relativement faibles, elle retarde l'emprésurage. Elle donne un caillé mou qui se brise facilement. La synérèse est retardée et dans le rompage du caillé, il n'est pas possible d'obtenir des petits morceaux aussi fermes qu'avec le lait normal. Il y aura des pertes au rompage, le sérum sera laiteux et les rendements seront diminués. Dans la fabrication des fromages à pâte cuite, le "ressuyage " sera mauvais.

L'épreuve du Iab. - On a recommandé l'épreuve du lab pour reconnaître si un lait est ou non malade. Cette épreuve est, à n'en pas douter, intéressante, et elle peut se recommander dans les fromageries importantes et dans les écoles de fromagerie, ne serait-ce que pour montrer les conséquences au point de vue de l'emprésurage d'une certaine proportion de lait malade dans une quantité plus importante de lait normal. Elle aura également cet avantage de mettre en relief l'influence nocive du colostrum et des laits de rétention, laquelle est due pour une bonne part à leur richesse en globuline.

Mais la reconnaissance de l'infection du lait peut déjà se faire par des procédés plus simples. A côté de l'augmentation de la globuline on doit noter une augmentation de l'azote non protéique : urée, acides aminés, etc. Son dosage, important au point de vue scientifique, est d'un intérêt moindre sur le terrain de la pratique. 


\section{L'ANAL YSE DES LAITS MALADES. CE QU'IL FAUT EN PRENDRE.}

Nous venons de donner l'aspect chimique des modifications essentielles que l'infection imprime au lait normal. Examinons-en maintenant les conséquences analytiques d'ordre chimique, physicochimique et biochimique, puis nous verrons celles qui se rattachent aux modifications portant sur les ferments et les leucocytes.

LE DOSAGE DU LACTOSE. - Nous apprécierons la diminution du taux du lactose par des méthodes différentes. Le dosage par la liqueur de Fehling nous donnera le chiffre exact du lactose. C'est une méthode chimique courante et sur laquelle nous n'avons pas à insister. Disons seulement qu'elle appartient au laboratoire.

L'indice de réfraction des sérums des laits malades. Mais le lactose est également le facteur principal de l'indice de réfraction du sérum du lait; si bien que mesurer cet indice, c'est en quelque sorte doser le lactose. La précision ici n'est pas celle que l'on obtient par le dosage direct avec la liqueur de Fehling, mais elle est suffisante pour la grande pratique.

La réfractométrie. - L'instrument que nous utiliserons est le réfractomètre. Il nous permet de faire des déterminations en série. Il y a longtemps que la grande industrie laitière utilise le réfractomètre dans la recherche $d u$ mouillage. Le mouillage n'étant qu'une dilution du lait par de l'eau fait diminuer le taux du lactose et la mesure de l'indice $n$ nous permet d'apprécier avec une exactitude assez grande l'importance de cette manœuvre dolosive.

Dans le cas qui nous occupe, il ne s'agit pas de mouillage, mais bien de dépister les laits malades. Le réfractomètre, en donnant la mesure de l'indice $\mathrm{n}$, nous fixe sur le taux du lactose et, par suite, nous permet d'apprécier l'étendue de l'altération du lait.

Lorsqu'il y a mouillage, la dilution porte sur tous les principes du lait quels qu'ils soient, aussi bien du chlorure que du lactose, et le jeu inverse du chlorure et du lactose que nous avons signalé tout à l'heure dans les laits malades n'est point constaté. Avec le mouillage, quand le lactose baisse, le chlorure baisse également, et dans les mêmes proportions.

LE DOSAGE DES CHLORURES. - Lorsqu'il s'agit de laits malades, si le sucre baisse le sel s'élève, comme nous le savons. Dans ees conditions, il importe de penser au dosage des chlorures. Le dosage des chlorures se fait par voie chimique. Des méthodes physiques ont bien été proposées, mais elles ne sauraient être utilisées ici. Nous ne nous étendrons pas sur la méthode à suivre, mais nous signalerons qu'en Allemagne on a mis à la portée du vétérinaire praticien une trousse qui lui permet de faire sur le terrain, peut-on dire, un dosage approximatif des chlorures. 
Si le sens du goût lui permet déjà de déceler la saveur saléeamère d'un lait suspect, il peut procéder à un dosage rapide des chlorures par la méthode qui utilise le virage du bichromate de potasse par formation de chromate d'argent, une fois que tout le chlore est précipité sous forme de chlorure d'argent. D'après les vétérinaires allemands, la méthode ne fournirait que $21 \%$ d'indications justes. Ces résultats sont certainement imputables, comme le dit A. TAPERNoux [38], au procédé recommandé par le fabricant de l'appareil.

La plus grande conductibilité des laits malades. L'excès de chlorures - et c'est toujours de chlorure de sodium qu'il s'agit - qu'on constate dans le lait malade, a une influence marquée sur une autre donnée physico-chimique du lait : la conductibilité, Celle-ci est due aux sels solubles du lait et, parmi eux, principalement aux chlorures. Toutes les fois que le taux des chlorures s'exagère, la conductibilité du lait s'élève, e'est-à-dire que sa résistance, qui en est à l'inverse, diminue. Mesurer la résistance électrique, 'c'est en quelque sorte déterminer indirectement les proportions de chlorure de sodium dans le lait. Un lait salé est nécessairement un lait dont la résistance électrique est moindre.

Des appareils ont été fabriqués par l'industrie pour permettre de déterminer facilement la résistance du lait. Ils sont tous basés sur l'établissement d'un pont de Wheastone, mais nous disposons de moyens encore plus simples pour fixer notre diagnostic.

LES LAITS MALADES VONT VERS L'ÁLCALINITE. - Nous avonS vu plus haut que l'infection, en diminuant le taux des phosphates du lait et notamment des phosphates potassiques, facteurs principaux de l'acidité, déterminaient en somme une marche du lait vers l'alcalinité.

Un lait est dit alcalin au point de vue titrimétrique lorsque, par titrimétrie, son degré Dornic est inférieur à 15,5-16, donnée normale inférieure d'un lait de grand mélange.

Il est dit alcalin au point de vue ionique lorsque son $p \mathrm{H}$, partant du $p \mathrm{H}$ moyen ( 6,5 environ), s'élève vers $7 ; 6,6$ est encore une donnée normale, 6,7 commence à être une donnée pathologique. Et plus nombreux qu'on ne le croit sont les laits dont le $p H$ est 6,8 , dépasse 7 et atteint même 7,2 et 7,3 .

L'emploi de l'acidimètre dans le but de procéder à une détermination d'acidité par titration avec la soude en présence de phtaléine du phénol demande du temps et un appareil sans doute simple, mais assez fragile ; c'est une raison pour laquelle nous ne la recommandons pas pour le dépistage des mammites. Mais ce n'est pas la seule. Ce n'est pas le lieu de nous étendre sur la comparaison de 
l'acidité actuelle et de l'acidité potentielle. Nous dirons simplement qu'elles ont des significations tout à fait différentes et que toutes les fois que nous pouvons apprécier l'acidité du lait par les méthodes qui relèvent de la détermination de l'acidité actuelle, e'est-à-dire de la concentration en ions $\mathrm{H}$, il faut le faire.

La determination de l'acidite actuelle. Le $p$ h. - La détermination du $p \mathrm{H}$ peut se faire par :

a) la méthode électrométrique ou

b) la méthode colorimétrique.

La méthode électrométrique. - La première appartient au laboratoire; elle se sert du potentiomètre, dont le maniement est simple. Toutefois ce potentiomètre ne saurait être utilisé couramment. Les indications qu'il donne sont plus précises que celles que nous fournit la colorimétrie, mais pour la grande pratique, celle-ci nous donne entièrement satisfaction.

La méthode colorimétrique. - Quatre indicateurs ont été recommandés pendant ces dernières années :

$1^{\circ}$ Le bleu de bromo-thymol;

$2^{\circ}$ L'alizarine ;

$3^{\circ}$ L'acide rosolique ;

$4^{\circ}$ Le pourpre de bromo-crésol.

Avec le bleu de bromo-thymol, le lait normal ne donne qu'une réaction jaunâtre faible amorcée vers le vert. Dès que la teinte verte apparaît franchẹment, et elle s'exagère avec le lait nettement alcalin, nous sommes dans la zone suspecte.

On a lancé récemment dans le commerce l'emploi d'un réactif : le thymobrol, qui n'est autre, sous ce nom spécial, qu'une solution de bleu de bromo-thymol. ROEDER [39] en a même recommandé l'emploi associé à l'épreuve catalasimétrique.

Les variations de teintes avec l'emploi du bleu de bromo-thymol s'étalent entre les deux teintes principales : le jaune du côté acide, le vert du côté alcalin. La nuance de la couleur obtenue nous indique dans quelle zone du $p \mathrm{H}$ nous nous trouvons.

Avec le pourpre de bromo-crésol, les virages au delà desquels, d'un côté comme de l'autre, aucune différenciation entre les teintes ne puisse être faite, vont d'un $p \mathbf{H}=5,2$ à $p H=6,8$; c'est dire qu'il s'agit surtout d'un indicateur des laits acides et de ceux dont l'alcalinité débute.

L'acide rosolique avait été recommandé par notre confrère Hö̈BERG. Il donne une couleur jaune orange pour le lait sain et rose carmin pour le lait nettement alcalin. Entre la première et la dernière de ces teintes, s'en étagent plusieurs plus ou moins rouge saumoné que la grande pratique permet d'apprécier. 
L'ÉREUVE A L'ALIZARINE. - II nous semble que ces indicateurs colorants ont à céder le pas devant l'alizarine. L'emploi de l'alizarine a été recommandé il y a plusieurs années par MoRres [40]. II s'agit d'une solution saturée d'alizarine dans l'alcool à $70^{\circ}$, alcalinisée ensuite avec beaucoup de précaution de façon que sa te.nte scit celle du vieux bordeaux. C'est exactement celle de la solution que je vous présente. Elle est facile à obtenir et il faut s'y tenir rigoureusement. Dans le commerce, on a lancé des solutions d'alizarine trop violacées et comme le volume du réactif à employer est égal ici à celui du lait, un réactif dont la teinte serait mal réglée donnerait avec le lait un virage qui ne correspondrait peut-être pas au $p H$ réel de ce liquide.

La solution d'alizarine se fait, comme nous venons de le dire, dans de l'alcool à $70^{\circ}$.

L'épreuve à l'alcool. - Déjà l'alcool pur à $70^{\circ}$ est employé depuis longtemps dans la grande industrie laitière pour reconnaître les laits acides. Un lait normal mélangé à son volume d'alcool à $70^{\circ}$ ne doit pas perdre sa fluidité. Le moindre grumeau qui apparaît est signe d'une acidité commençante et, par conséquent, le lait doit être mis de côté.

L'épreuve se fait rapidement. On mélange avec des pipettes ad hoc $2 \mathrm{~cm}^{3}$ de lait à $2 \mathrm{~cm}^{3} \mathrm{~d}^{\prime}$ alcool à $70^{\circ}$ dans un tube et on renverse avec précaution, de façon à pouvoir examiner la nappe liquide qui s'écoule. La moindre floculation fait éliminer le lait pour l'alimentation en nature ; c'est du lait qui coagulerait lors de la pasteurisation.

Beaucoup de travaux ont été entrepris sur cette épreuve dite épreuve à l'alcool parce qu'on s'était aperçu que certains laits normaux coagulaient cependant à $70^{\circ}$. Lorsqu'on chercha à pénétrer dans le mécanisme de la réaction, on se rendit compte que l'épreuve à l'alcool est moins une épreuve d'acidité qu'une épreuve d'équilibre salin. Si celui-ci est troublé, surtout dans le sens d'un excès, si faible soit-il, de sel de calcium soluble, on obtiendra une coagulation, alors même que l'acidité traduite titrimétriquement et potentiométriquement reste dans la normalité.

Les recherches que nous sommes en train de faire dans notre laboratoire sur cette question nous mettent dans l'obligation d'attribuer à la stabilité des équilibres salins du lait la plas grande importance pour juger de l'épreuve à l'alcool. La moindre déviation, comme nous disions tout à l'heure, en faveur des sels de calcium solubles rend la réaction positive. L'acidification du lait intervient en solubilisant de la chaux colloidale, en augmentant par suite le taux des sels vraiment solubles de calcium, des sels cristalloïdes.

Toutefois ces réserves étant faites, il nous faut reconnaître que l'épreuve à l'alcool a déjà l'avantage de sa rapidité. On doit lui accorder une certaine confiance lorsqu'il s'agit justement des laits 
de ramassages, moyens et grands ramassages, destinés à la consommation en nature. Mais pour des laits individuels, sa valeur peut être critiquée.

La réaction à l'alizarol doit être considérée sous deux aspects : par son côté alcool et par son côté coloré.

Par son côté alcool, elle nous donnera les mêmes indications que 1'alcool pur à $70^{\circ}$.

Par son côté coloré, elle nous en fournit d'autres qui sont de la plus haute importance dans le dépistage de l'infection latente. Nombreux, en effet, sont les auteurs qui reconnaissent à ce réactif la plus grande valeur.

Avec K. KatrandjiefF [41] [42], nous l'avons utilisé sur des laits provenant des abattoirs de Lyon. Les uns étaient quelquefois normaux, mais la plupart étaient des laits de rétention pure, la mamelle n'étant pas très infectée, et des laits mixtes, c'est-à-dire de rétention et d'infection tout à la fois.

Ces laits étaient en général alcalins et les colorations que nous obtenions le plus souvent s'étalaient, en effet, depuis le rouge violacé jusqu'au violet foncé ( $p H$ allant de 6,8 à 7,3 ).

La gamme des violets est extrêmement variable. Elle est faite de nuances auxquelles la grande pratique vous permettra rapidement de donner une signification exacte. L'échelle des teintes est beaucoup plus variée que celle que l'on trouve dans l'utile travail de Valencien et Panchadd [43] sur la question. C'est surtout sur la série des tons rouges violacés et violets que nous tenons à appeler votre attention en $\mathrm{y}$ attachant le $p \mathrm{H}$ qui leur correspond.

A quoi sont dus les flocons dans la simple épreuve à l'alcool sur les laits acides? - Lorsqu'on fait la simple épreuve à l'alcool à $70^{\circ}$ sur des laits à tendance acide - et c'est ceux-là que vise la grande industrie laitière, dont le but est d'alimenter les villes en lait -, on observe, à partir d'un certain moment, en même temps qu'une variation de teinte vers la translucidité due à l'apparition du sérum, la production de flocons. L'explication nous en est maintenant connue. Il s'agit de caséine ou plutôt de caséinate, dont la précipitation est facilitée par l'alcool à $70^{\circ}$ en présence d'un excès de chaux soluble dû, pour commencer, à l'action de l'acide lactique sur certains sels de calcium existant à l'état colloidal, tels les phosphates.

(A suivre.) 